\title{
(2) OPEN ACCESS \\ Real world evidence on 5661 patients treated for macular oedema secondary to branch retinal vein occlusion with intravitreal anti-vascular endothelial growth factor, intravitreal dexamethasone or macular laser
}

\author{
Richard Gale, ${ }^{1,2}$ Maria Pikoula, ${ }^{3}$ Aaron Y Lee, ${ }^{4}$ Spiros Denaxas, ${ }^{3}$ Catherine Egan, ${ }^{5}$ \\ Adnan Tufail, ${ }^{5}$ Paul Taylor ${ }^{0},{ }^{3}$ on behalf of UK EMR Users Group
}

1 Ophthalmology, Hightown Surgery, York, UK

${ }^{2}$ York Teaching Hospital NHS Foundation Trust, York, UK ${ }^{3}$ Institute of Health Informatics, University College London, London, UK

${ }^{4}$ Department of Ophthalmology, University of Washington, Seattle, Washington, USA ${ }^{5}$ Moorfields Eye Hospital NHS Foundation Trust, London, UK

Correspondence to

Dr Paul Taylor, Institute of Health Informatics, University College London, London WC1E 6BT, UK; p.taylor@ucl.ac.uk

Received 10 January 2020 Revised 27 April 2020 Accepted 14 May 2020

\section{Check for updates}

(c) Author(s) (or their employer(s)) 2020. Re-use permitted under CC BY-NC. No commercial re-use. See rights and permissions. Published by BMJ.

To cite: Gale R, Pikoula M, Lee AY, et al. Br J Ophthalmol Epub ahead of print: [please include Day Month Year]. doi:10.1136/

bjophthalmol-2020-315836

\begin{abstract}
Background/aims Clinical trials suggest anti-vascular endothelial growth factor is more effective than intravitreal dexamethasone as treatment for macular oedema secondary to branch retinal vein occlusion. This study asks if 'real world' data from a larger and more diverse population, followed for a longer period, also support this conclusion.

Methods Data collected to support routine care at 27 NHS (National Health Service) Trusts between February 2002 and September 2017 contained 5661 treatment-naive patients with a single mode of treatment for macular oedema secondary to branch retinal vein occlusion and no history of cataract surgery either during or recently preceding the treatment. Number of treatment visits and change in visual acuity from baseline was plotted for three treatment groups (anti-vascularendothelial growth factor (anti-VEGF), intravitreal dexamethasone, macular laser) for up to 3 years.
\end{abstract}

Results Mean baseline visual acuity was 57.1/53.1/62.3 letters in the anti-VEGF/dexamethasone/ macular laser groups, respectively. This changed to 66.72 $(+9.6) / 57.6(+4.5) / 63.2(+0.9)$ at 12 months. Adequate numbers allowed analysis at 18 months for all groups $(66.6(+9.5) / 56.1(+3.0) / 60.8(-1.5))$ and for anti-VEGF at 36 months $(68.0,+10.9)$ Mean number of treatments were 5.1/1.5/1.2 at 12 months, 5.9/1.7/1.2 at 18 months for all three groups and 10.3 at 36 months for anti-VEGF.

Conclusions Visual acuity improvements were higher and more sustained with anti-VEGF. Higher treatment burden occurred with anti-VEGF but this reduced over 36 months. Patients with better vision at baseline than those in the clinical trials maintained high levels of vision with both anti-VEGF and dexamethasone.

\section{INTRODUCTION}

Branch retinal vein occlusion (BRVO) is a common cause of vision loss with an incidence of between $0.5 \%$ to $1.2 \% .^{1}$ Five per cent of patients present with bilateral disease and 10\% develop disease in both eyes within 10 years of the initial diagnosis. If untreated BRVO leads to significant loss of vision, only $50 \%$ to $60 \%$ of untreated eyes will retain visual acuity of $6 / 12$ or more after 1 year. $^{2}$

Secondary macular oedema (MO) is the most common cause of visual loss in BRVO. Five to fifteen per cent of patients develop MO within 12 months of diagnosis and 50\% of these suffer visual loss. ${ }^{3}$ Three principle treatment options for MO secondary to BRVO are discussed in the Royal College of Ophthalmologists' guidelines: macular laser, intravitreal injections of antivascularendothelial growth factor (anti-VEGF) and steroid implants. ${ }^{4}$

Of the three treatments, macular laser was the first to be shown to be effective, when, in 1986, the Branch Retinal Vein Occlusion Study demonstrated that, at 36 months, 28 of 43 (63\%) participants who had macular laser for MO gained two or more lines of vision on a Snellen chart compared with 13 $(37 \%)$ who were untreated. ${ }^{2}$

The first evidence of the effectiveness of both anti-VEGF and steroid implants was published in reports of two separate trials in 2011. In the BRAVO study, ranibizumab-an anti-VEGF treatment-was given monthly for six doses and then as required for the following 6 months. Patients achieved a mean gain of 18.3 letters at month 6 with stability thereafter using a mean of 5.7 injections in the first 6 months and 2.7 in the second 6 months. ${ }^{5}$ Twenty per cent of patients received macular laser at month 5 in accordance with the trial protocol for suboptimal response. The GENEVA study programme evaluated the use of the intravitreal steroid implant dexamethasone (Ozurdex) for RVO. The subgroup of patients diagnosed with BRVO achieved a peak mean gain of 10 letters and had a mean ETDRS letter gain of six letters. ${ }^{6}$ Retreatment was allowed at 6 months and the benefit at 12 months was again six letters. Further trials of both treatments, including other anti-VEGF agents, followed. In the VIBRANT study, patients given monthly injections of aflibercept achieved a mean gain of 17 letters at 6 months with a mean of 5.7 injections.

The 2015 RVO guidelines state that 'although any of these drugs may be used as first line for this condition, anti-VEGF is preferred in eyes with a previous history of glaucoma and younger patients who are phakic. Ozurdex may be a better choice 
in patients with recent cardiovascular events and in those who do not favour monthly injections'. ${ }^{4}$ More recently head-tohead evidence comparing the two categories of treatment has become clearer and a recent meta-analysis identified four trials assessing 643 eyes with BRVO and found that anti-VEGF treatments showed a greater improvement in visual acuity than the dexamethasone implants: a mean difference between treatments of 9.25 letters at 6 or 12 months. ${ }^{8}$

In addition to these clinical trials, some limited analyses of 'real world evidence' have been published. For example, Rezar et al followed 14 patients treated for BRVO with ranibizumab and found an improvement from a mean of 44 to 76 letters at longterm follow-up. ${ }^{9}$ Wecker et al present data on 351 eyes treated with anti-VEGF for RVO and showed that a stable percentage of the number being treated had a $>15$ letter gain: $24.2 \%$ (1 year), $26.3 \%$ ( 2 years) and $24.2 \%$ (5 years), but note that the number being treated (and therefore recorded in the data set) drops to 198 at year 1 and 33 at year $5 .{ }^{10}$ Chatziralli et al analysed routine data from 29 BRVO patients treated with ranibizumab, with a mean visual acuity (VA) of 63.7 at baseline, and found improvements to 74.9 at 12 months and 77.4 at 24 months. ${ }^{11}$ Spooner et al followed 31 eyes treated for BRVO with anti-VEGF for 5 years and found a mean change in VA of +14.1 letters. ${ }^{12}$ We found only one study that compared anti-VEGF and other treatments using real world data, that of Winterhalter et al who present data on visual acuity after 6 months of treatment with ranibizumab $(n=58)$ or dexamethasone $(n=48)$ and found improvements in the mean visual acuity of both groups (from 59.5 to 67 and from 54.5 to 65.5$).^{13}$

The UK EMR Users group has previously analysed data obtained from an electronic medical record (EMR), first looking at patients with age-related macular degeneration and then patients with diabetic macular oedema. They found that real world visual outcomes were not as good as those in clinical trials but that the studied treatments were nevertheless effective, and effective in wider populations than were included within the criteria of relevant trials. ${ }^{1415}$ This study evaluates the effectiveness and treatment burden of anti-VEGF therapy, intravitreal dexamethasone and macular laser for BRVO, in the same large data set, that of the UK EMR Users group, identifying a much larger group of patients than earlier studies. We seek to complement the evidence that has emerged from clinical trials, asking three questions:

1. Do patients treated outside of clinical trials have the same positive outcomes reported in relevant trials?

2. Do patients who are outside the typical trial inclusion criteria benefit from treatment?

3. Are the benefits sustained after the period for which trial patients were followed up?

We also looked at data on what proportion of patients switched between the three recommended treatments, and at what point in treatment they switched.

\section{METHODS}

\section{Study design and inclusion criteria}

Twenty-seven sites making comprehensive use of the Medisoft EMR system to record ophthalmology treatments agreed to contribute data to studies of retinal diseases, including RVO. Data were recorded between 01 February 2002 and 03 September 2017. Length of follow-up varied depending on when the patient was first entered onto the system and for how long the patient's treatment and VA assessments were recorded.

\section{Population}

All patients recorded as receiving treatment for 'branch retinal vein occlusion' were included. Patients with missing data for age and gender were excluded. Patients who were not treatment

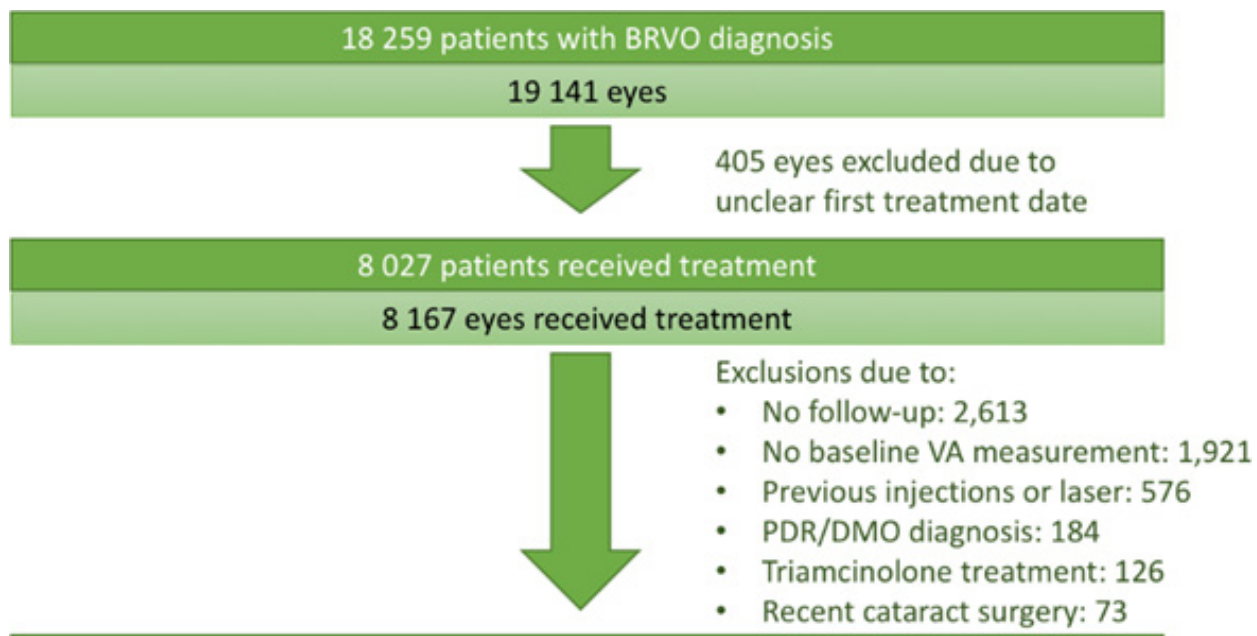

5188 patients treatment naive with baseline and follow-up VA measurements

5251 eyes treatment naïve with baseline follow-up VA measurements

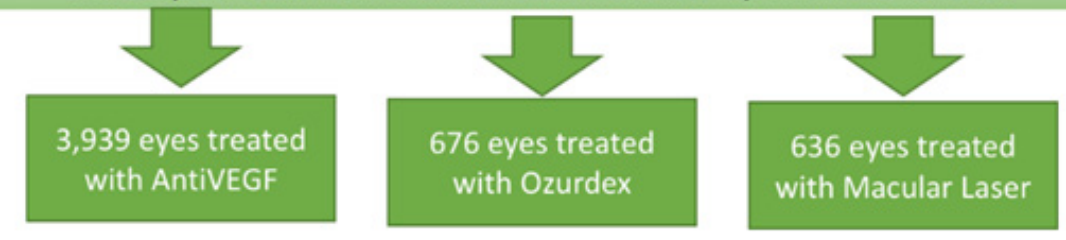

Figure 1 ConsolidatedStandards of Reporting Trials flow diagram for the entire patient population. anti-VEGF,anti-vascular endothelial growth factor; BRVO, branchretinal vein occlusion; DMO, diabetic macular oedema; PDR, proliferativediabetic retinopathy; VA,visual acuity. 
naïve or were receiving one of the treatments of interest for another condition were excluded, for example, patients with proliferative diabetic retinopathy or "clinically significant macular oedema' were excluded as it could not be certain that the MO for which these patients were being treated was secondary to BRVO. Patients with a record of cataract surgery in the 3 months before the first treatment for BRVO were also excluded. Patients who had been flagged as receiving treatments as part of a blinded randomised trial were also excluded. Any event where data was missing was also excluded. Patients receiving intravitreal triamcinolone were excluded because of the 126 eyes initiated on this treatment only 24 met our inclusion criteria.

The date of exit from the study for a patient was defined as the date of either the last VA measurement or the last VA measurement prior to switching treatment modality or receiving cataract surgery.

Patients were divided into three cohorts according to the treatment received: (1) the 'anti-VEGF' cohort including ranibizumab (Lucentis), and aflibercept (Eylea); (2) the 'dexamethasone' (Ozurdex) cohort and (3) the 'macular laser' cohort.

Data on age (years) and ethnicity were extracted from the local patient administration system. Social deprivation was assessed using the 2015 Index of Multiple Deprivation for all patients and stratified in quintiles. ${ }^{16}$

\section{Visual acuity measurement}

Visual acuity was measured as a part of routine clinical practice and recorded as an ETDRS score, Snellen or LogMar acuity measurement. All numerical measurements were converted to an ETDRS letter score using a standard algorithm. ${ }^{17} \mathrm{~A}$ number of eyes were recorded as having baseline vision of 'counting fingers' or 'hand movements'. Numbers in these categories are reported but these eyes are excluded from numerical analyses using pooled VA measurements. Eyes where VA measurements decreased to 'counting fingers' or lower levels were given a value of 0 when calculating mean ETDRS scores.

\section{Statistical analysis}

To compare the effectiveness of the three treatment modalities, measurements of absolute VA and change from baseline visual acuity were plotted for each of the three treatment groups for all patients over 3 years from baseline at approximately monthly intervals. Figures for mean VA and mean changes in VA each month from baseline were calculated using data only from the patients measured in that month, no interpolated data was used in calculations, but in the patients' trajectories are illustrated in the figures by carrying forward individuals' measured VA until the next measurement. The impact of length of follow-up on outcome was assessed by charting the VA measurements over time separately for three cohorts defined by length of follow-up.

Numbers were sufficiently high in the anti-VEGF group to allow assessment of the impact of age and VA at baseline by stratification into quintiles. Kaplan-Meier figures were plotted to show time to treatment switching and time to 15 letter loss. Treatment burden was evaluated using number of visits and number of injections.

Signed permission to analyse anonymised data was returned from the lead clinician and Caldicott guardian (the clinician responsible for data protection in an NHS (National Health Service) trust) at each participating trust. Fully anonymised data was extracted on 11 December 2017.
Table 1 Demographic information and mean visual acuity at baseline and 6, 12, 18, 24 and 36 months (the number of eyes with a measurement at each point is shown in parentheses). Data on treatment burden and the numbers and percentages of eyes switching treatment are also shown

\begin{tabular}{|c|c|c|c|}
\hline & Anti-VEGF & Macular laser & Dexamethasone \\
\hline Mean age at baseline & 72.0 & 72.5 & 71.9 \\
\hline Gender (\% male) & 47.4 & 48.6 & 45.9 \\
\hline Baseline CF (\%) & $57(1.3)$ & $14(2.2)$ & $14(2.1)$ \\
\hline Baseline HM (\%) & $23(0.6)$ & $2(0.3)$ & $3(0.4)$ \\
\hline \multicolumn{4}{|c|}{ Mean VA in ETDRS letters ( $n$ ) } \\
\hline At baseline & 57.1 (3859) & $62.3(620)$ & $53.1(659)$ \\
\hline At 6 months & 65.9 (1598) & $60.9(110)$ & 59.7 (188) \\
\hline At 12 months & $66.7(1042)$ & $63.2(53)$ & $57.6(95)$ \\
\hline At 18 months & $66.6(713)$ & $60.8(48)$ & $56.1(71)$ \\
\hline At 24 months & $67.2(485)$ & $56.4(30)$ & $59.3(45)$ \\
\hline At 36 months & $68(203)$ & $50.1(23)$ & $62.9(20)$ \\
\hline
\end{tabular}

anti-VEGF, anti-vascular endothelial growth factor; $C F$, counting fingers; $H M$, hand movement; VA, visual acuity.

\begin{tabular}{|c|c|c|c|c|c|c|}
\hline & \multicolumn{2}{|c|}{ Anti-VEGF } & \multicolumn{2}{|c|}{ Macular laser } & \multicolumn{2}{|c|}{ Dexamethasone } \\
\hline & Visits & Treatments & Visits & Treatments & Visits & Treatments \\
\hline \multicolumn{7}{|c|}{ Mean total visits and treatment visits (per patient) } \\
\hline At 6 months & 5.2 & 3.8 & 2.3 & 1.1 & 3.9 & 1.3 \\
\hline At 12 months & 7.9 & 5.1 & 3.2 & 1.2 & 5.6 & 1.5 \\
\hline At 18 months & 9.5 & 5.9 & 3.8 & 1.2 & 6.6 & 1.7 \\
\hline At 24 months & 10.6 & 6.4 & 4.2 & 1.2 & 7.2 & 1.7 \\
\hline At 36 months & 11.7 & 6.9 & 4.6 & 1.2 & 7.8 & 1.7 \\
\hline \multicolumn{7}{|c|}{ Treatment switching (\%) } \\
\hline To anti-VEGF & \multicolumn{2}{|l|}{-} & \multicolumn{2}{|c|}{$80(12.6)$} & \multicolumn{2}{|c|}{$285(42.2)$} \\
\hline To macular laser & \multicolumn{2}{|c|}{$267(6.8)$} & \multicolumn{2}{|l|}{-} & \multicolumn{2}{|c|}{$63(9.3)$} \\
\hline $\begin{array}{l}\text { To } \\
\text { dexamethasone }\end{array}$ & \multicolumn{2}{|c|}{$162(4.1)$} & \multicolumn{2}{|c|}{$83(13.0)$} & \multicolumn{2}{|l|}{-} \\
\hline
\end{tabular}

anti-VEGF, anti-vascular endothelial growth factor.

\section{RESULTS}

Of the 19141 eyes with a recorded diagnosis of BRVO, 5251 were included in the study, reasons for exclusion are listed in the Consolidated Standards of Reporting Trials diagram in figure 1. The mean age of the study population was 72.1 years, and $47.4 \%$ of patients were male. Ethnicity was recorded for only $64 \%$, of those $87 \%$ were recorded as White. All quintiles of social deprivation were approximately equally represented.

\section{Visual acuity}

The age, gender and mean VA of the three treatment groups at baseline and 6, 12, 18, 24 and 36 months in ETDRS letters, are shown in table 1 .

Patients were lost to follow-up (the reasons for which are not recorded in the data) at a roughly constant rate, starting immediately after baseline with a peak at 4 months. The mean period for which data was recorded on a patient was 1.4 years. The numbers in the cohort therefore fall rapidly, with 1090 patients having a measurement at 12 months and 560 and 246 at 24 and 36 months, respectively. Only 15 patients have 5 years or more of data. Table 1 also illustrates treatment burden across the whole population over 36 months with a mean of 10.3 visits ranging from a mean of 4.6 to 11.7 visits depending on treatment type. Sixty-three (1.2\%) of the 5188 patients in the study had a second eye treated (anti-VEGF: 45, laser: 9, dexamethasone: 9). Patients were included in the study until either the last recorded VA measurement or the last recorded measurement 

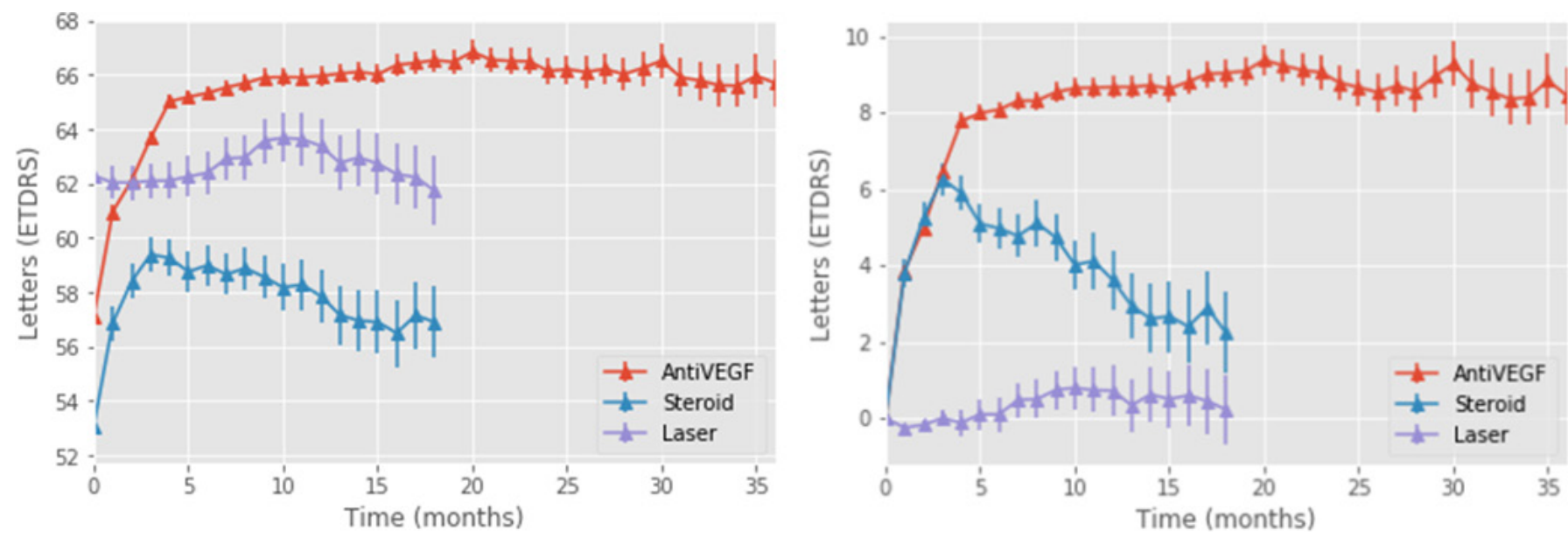

Figure 2 Mean VA (left) and change from baseline in VA (right) during the first 3 years for 215 all treatment groups. anti-VEGF,anti-vascular endothelial growth factor; VA, visualacuity.

before the patient switched treatment or received cataract surgery. Numbers of patients switching between the different treatments are given in table 1.

Figure 2 shows the mean VA and absolute change in VA from baseline in the three groups, defined by treatment type. Figure 3 shows the mean VA and absolute change in VA from baseline for the study population stratified by baseline VA quintiles. Figure 4 illustrates the difference in VA measurements over time for patients above and below the upper threshold for inclusion in the VIBRANT and GENEVA studies.

\section{DISCUSSION}

This study is the largest to report on a cohort of UK patients with BRVO. It provides detailed information about outcomes and treatments of 5188 patients, with 246 patients followed up for 3 years. The demographic data show that the patients in the three treatment groups were of similar age, and had similar mixes of gender, ethnicity and similar scores for social deprivation. The mean baseline visual acuity was higher for patients treated with macular laser and lower for patients treated with dexamethasone. It is possible that clinician or patient choice of treatment is influenced by the perceived severity of the occlusion, with less severe being offered macular laser. To assess whether the difference in outcomes between patients treated with
anti-VEGF and dexamethasone was due to severity of disease we compared baseline central retinal thickness of eyes treated with anti-VEGF $(n=249$, mean $=529 \mu \mathrm{m})$ or dexamethasone $(n=110$, mean $=515 \mu \mathrm{m})$, where the measurement was recorded on a Heidelberg Spectralis machine (only very small numbers of eyes had measurements recorded using other devices): the difference is not statistically significant $(\mathrm{p}=0.44)$.

Relative gains in VA for patients treated with anti-VEGF were lower than in key clinical trials: 11.5 at 12 months compared with 18.4 in the $0.5 \mathrm{mg}$ ranibizumab cohort in BRAVO. Fewer injections were given in this study. Patients in the anti-VEGF cohort had a mean of 5.1 injections in the first 12 months. This partly reflects that many more patients in this study dropped out, but the figure is still only 6.0 when patients who dropped out before 12 months are excluded. This compares to 8.5 over 12 months in BRAVO and 9.0 in the first 48 weeks of VIBRANT. Mean baseline VAs in these studies was comparable at 56 ETDRS letters for BRAVO and 59 for the treatment group in VIBRANT, compared with 57.1 in this cohort.

Gains in VA with dexamethasone at 12 months $(+4.5)$ fell short of trial outcomes, using patients in the GENEVA study for comparison $(+6)$. This may be because baseline VA was 57.7 in this cohort compared with 54 in GENEVA and fewer treatments were given over 12 months, 1.5 compared with two for
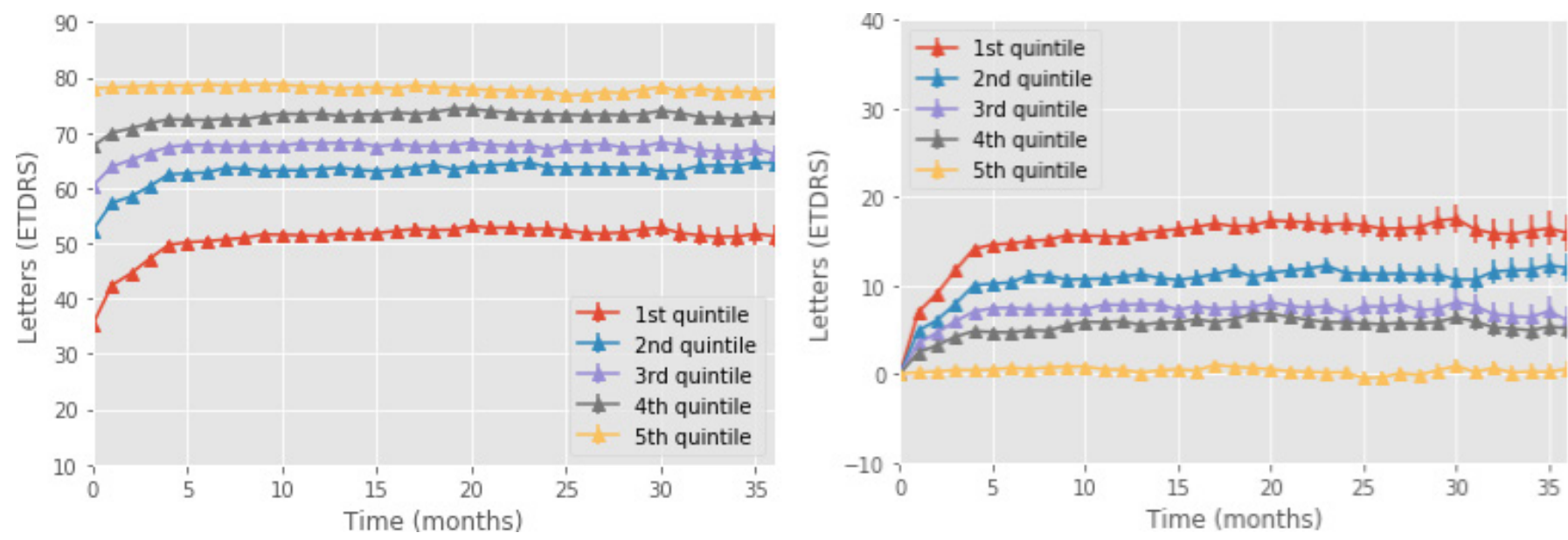

Figure 3 Mean (left) and change (right) in VA stratified by baseline VA for the entire cohort. Percentage of patients at baseline with a measurement at 36 months for each quintile is: Q1: 4.6\%, Q2: 7\%, Q3: 4.7\%, Q4: 5.2\%, Q5: 5\%. VA, visual acuity. 

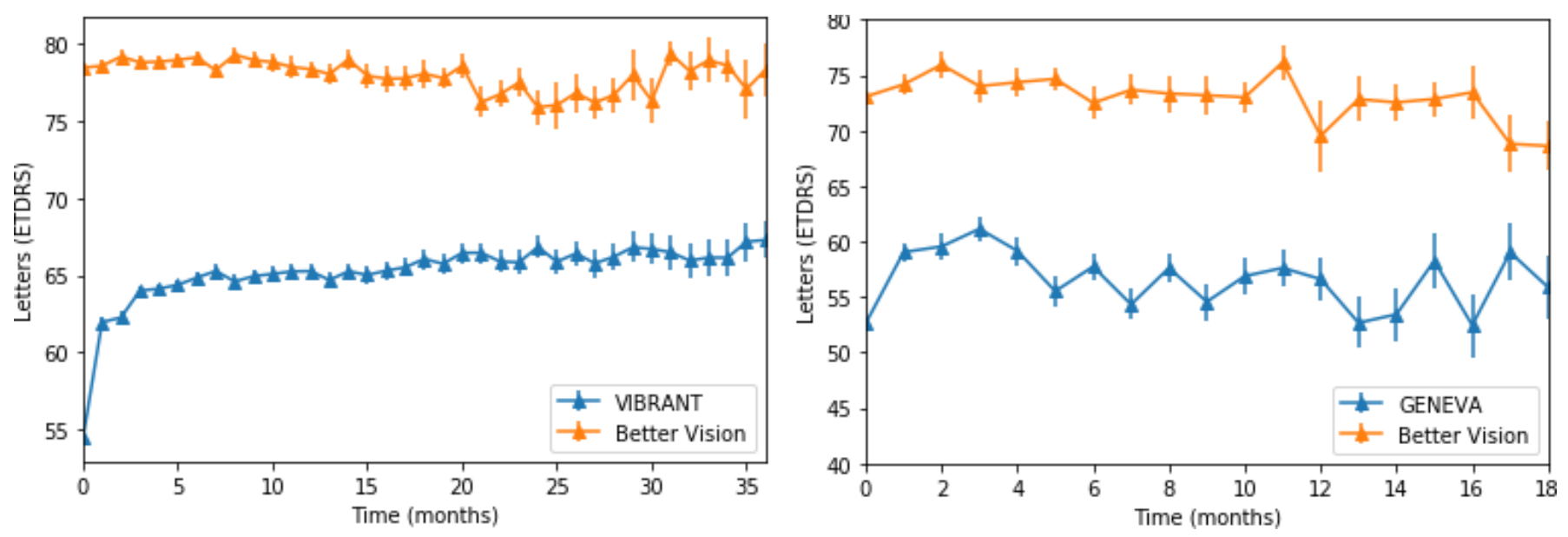

Figure 4 Mean in visual acuity for patients in our study who met the inclusion criteria for the VIBRANT and GENEVA trials, compared with that for patients with baseline visual acuity above the level set in each trial.

the retreatment group in GENEVA. ${ }^{6}$ It should also be noted that more recent experience with dexamethasone suggests that retreatment before 6 months improves outcomes. ${ }^{18}$

Possible explanations of the reduced effectiveness include different case selection and dropout, fewer treatments and the effect of higher starting visual acuity. A key outcome of the study is that although patients with the lowest baseline VA show the greatest absolute improvement, patients treated with the highest baseline VA have the best outcomes. Patients with worse starting VA did not reach that of those with better starting acuity, an effect also seen with treatment of neovascular age-related macular degeneration(nvAMD), indicating that early access to treatment is beneficial. ${ }^{14}$ As is clearly shown in figure 4 , patients with baseline VA that was above the threshold used for inclusion in the VIBRANT and GENEVA trials (and in the head-to-head comparisons of anti-VEGF and steroid injections reviewed by Gao L, Zhou L, Tian C, et $a l^{8}$ ) had better outcomes than those included in the trials.

Our study provides data on the long-term consequences of treatment with BRVO, longer than has been reported in any of the clinical trials to date. Patients clearly maintain vision longer when treated with anti-VEGF than with either intravitreal steroids or with macular laser.

Patients were more likely to improve when treated with anti-VEGF than with the alternative treatments. The cohort of patients treated with laser or with dexamethasone either did not improve or failed to maintain their improvement. It is important to appreciate that this may in part be because the three treatment cohorts start with different mean visual acuities. This effect is not however, related to the treatment for cataracts since these patients were excluded.

The burden of visits and of treatment is greater with antiVEGF than alternatives, but this has to be considered in the context of the better outcomes. It is worth noting that the additional number of visits required is lower in later years.

The proportion of patients with severe visual loss ('counting fingers' or less) is considerably lower in the group treated with anti-VEGF compared with both alternatives.

This study uses real world data and has the advantages and disadvantages of studies reporting on the routine care received by patients outside of the controlled environment of clinical trials. A particular difficulty is that of missing data. Not all patients are assessed for visual acuity at the same point in time, many patients are lost to follow-up and there is no way to determine whether the data is censored because of patients moved, declined further treatment or died. It should be noted that the shift in the mean VA measurement over time may not reflect the trajectories of individual patients but the changing composition of the cohort as more and more patients are progressively lost to follow-up or switched to alternative therapies.

This is largest study yet in the UK for patients with this condition and the outcomes should help inform clinician and patient choice and the planning of services. It shows that patients treated with the highest baseline VA have the best outcomes, presenting a strong argument for the prompt initiation of treatment.

\section{Twitter Paul Taylor @paul3548}

Collaborators UK EMR Users Group: Ms T Akerele, Hinchingbrooke Health Care NHS Trust; Mr R Antcliff, Royal United Hospital Bath NHS Trust; Miss C Bailey, University Hospitals Bristol NHS Foundation Trust; Mr C Brand, Sheffield Teaching Hospitals NHS Foundation Trust; Professor U Chakravarthy, Belfast Health and Social Care Trust; Miss A Davis, Moorfields Eye Centre at Croydon University Hospital; Mr N Dhingra, Mid Yorkshire Hospitals NHS Trust; Miss L Downey, Hull and East Yorkshire Hospitals NHS Foundation Trust; Mr H Eleftheriadis, King's College Hospital NHS Foundation Trust; Miss S George, The Hillingdon Hospital NHS Foundation Trust; Mr F Ghanchi, Bradford Teaching Hospitals NHS Foundation Trust; Mr C Jones, Norfolk and Norwich University Hospitals NHS Foundation Trust; Mrs R Khan, Calderdale and Huddersfield NHS Foundation Trust; Mr V Kumar, Wirral University Teaching Hospital NHS Foundation Trust; Mrs P Lip, Sandwell and West Birmingham Hospitals NHS Trust; Mr A Lobo, Moorfields Eye Centre at Bedford Hospital; Professor A Lotery, University Hospital Southampton NHS Foundation Trust; Mr S Mahmood, Central Manchester University Hospitals NHS Foundation Trust; Professor G Menon, Frimley Park Hospital NHS Foundation Trust; Mr R Mukherjee, Leeds Teaching Hospitals NHS Trust; Mr S Natha, Wrightington, Wigan and Leigh NHS Foundation Trust; Miss H Palmer, University Hospitals Birmingham NHS Foundation Trust; Miss S Patra, Bart's Health NHS Trust; Mr A Patwardhan, Royal Cornwall Hospitals NHS Trust; Mr B Paul, Barking, Havering and Redbridge University Hospitals NHS Trust; Mr J Talks, The Newcastle Upon Tyne Hospitals NHS Foundation Trust; Dr E Wilkinson, Northern Devon Healthcare NHS Trust.

Contributors CE, RG, AL and AT designed the study. CE, PT and AT organised the collection of the data. The EMR Users Group provided the data. SD, AL and MP performed the analyses.

Funding This work was supported by a grant from Novartis Pharmaceuticals and received a proportion of its funding from the Department of Health's NIHR Biomedical Research Centre for Ophthalmology at Moorfields Eye Hospital and UCL Institute of Ophthalmology. The views expressed in the publication are those of the authors and not necessarily those of the Department of Health. Aaron Lee is supported by NIH/NEI K23EY029246 and an unrestricted grant from Research to Prevent Blindness.

Competing interests Paul Taylor received a grant from Novartis Pharmaceuticals. Adnan Tufail is a Consultant to Allergan, Bayer, Heidelberg Engineering, Kanghong Pharmaceuticals, Novartis and Oxurion. Catherine Egan is a Consultant to Heidelberg Engineering. Aaron Lee reports being an employee of the US Food and Drug 
Administration, grants from Santen, personal fees from Genentech, grants from Carl Zeiss Meditec, grants from Novartis, personal fees from Topcon, personal fees from Verana Health, outside the submitted work. This article does not reflect the opinions of the US Government or of the US FDA

Patient consent for publication Not required.

Ethics approval Appropriate institutional review board approval was obtained at the last named author's institution.

Provenance and peer review Not commissioned; externally peer reviewed.

Data availability statement Data may be obtained from a third party and are not publicly available. De-identified data was obtained from 27 participating trusts (names and contact details available on request) via Medisoft Limited.

Open access This is an open access article distributed in accordance with the Creative Commons Attribution Non Commercial (CC BY-NC 4.0) license, which permits others to distribute, remix, adapt, build upon this work non-commercially, and license their derivative works on different terms, provided the original work is properly cited, appropriate credit is given, any changes made indicated, and the use is non-commercial. See: http://creativecommons.org/licenses/by-nc/4.0/.

ORCID iD

Paul Taylor http://orcid.org/0000-0002-6296-2948

\section{REFERENCES}

1 Jaulim A, Ahmed B, Khanam T, et al. Branch retinal vein occlusion: epidemiology, pathogenesis, risk factors, clinical features, diagnosis, and complications. An update of the literature. Retina 2013:33:901-10.

2 Group BVOS. Argon laser scatter photocoagulation for prevention of neovascularization and vitreous hemorrhage in branch vein occlusion. A randomized clinical trial. branch vein occlusion Study Group. Arch Ophthalmol 1986;104:34-41.

3 Rogers SL, McIntosh RL, Lim L, et al. Natural history of branch retinal vein occlusion: an evidence-based systematic review. Ophthalmology 2010;117:1113-23.

4 Ophthalmologists TRCo. Retinal Vein Occlusion (RVO) Guidelines. The Royal College of Ophthalmologists, 2015.

5 Brown DM, Campochiaro PA, Bhisitkul RB, et al. Sustained benefits from ranibizumab for macular edema following branch retinal vein occlusion: 12-month outcomes of a phase III study. Ophthalmology 2011;118:1594-602.
6 Haller JA, Bandello F, Belfort R, et al. Dexamethasone intravitreal implant in patients with macular edema related to branch or central retinal vein occlusion twelve-month study results. Ophthalmology 2011;118:2453-60.

7 Clark WL, Boyer DS, Heier JS, et al. Intravitreal aflibercept for macular edema following branch retinal vein occlusion: 52-week results of the Vibrant study. Ophthalmology 2016;123:330-6.

8 Gao L, Zhou L, Tian C, et al. Intravitreal dexamethasone implants versus intravitreal anti-VEGF treatment in treating patients with retinal vein occlusion: a meta-analysis. BMC Ophthalmol 2019;19:1-10.

9 Rezar S, Eibenberger K, Bühl W, et al. Anti-VEGF treatment in branch retinal vein occlusion: a real-world experience over 4 years. Acta Ophthalmol 2015;93:719-25.

10 Wecker T, Ehlken C, Bühler A, et al. Five-Year visual acuity outcomes and injection patterns in patients with pro-re-nata treatments for AMD, DME, RVO and myopic CNV. Br J Ophthalmol 2017;101:353-9.

11 Chatziralli I, Theodossiadis G, Chatzirallis A, et al. Ranibizumab for retinal vein occlusion: predictive factors and long-term outcomes in real-life data. Retina 2018:38:559-68.

12 Spooner K, Fraser-Bell S, Hong T, et al. Five-Year outcomes of retinal vein occlusion treated with vascular endothelial growth factor inhibitors. BMJ Open Ophthalmol 2019;4:e000249.

13 Winterhalter S, Eckert A, Vom Brocke G-A, et al. Real-Life clinical data for dexamethasone and ranibizumab in the treatment of branch or central retinal vein occlusion over a period of six months. Graefes Arch Clin Exp Ophthalmol 2018:256:267-79.

14 Writing Committee for the UK Age-Related Macular Degeneration EMR Users Group. The neovascular age-related macular degeneration database: multicenter study of 92976 ranibizumab injections: report 1: visual acuity. Ophthalmology 2014;121:1092-101.

15 Egan C, Zhu H, Lee A, et al. The United Kingdom diabetic retinopathy electronic medical record users group, report 1: baseline characteristics and visual acuity outcomes in eyes treated with intravitreal injections of ranibizumab for diabetic macular oedema. Br J Ophthalmol 2017;101:75-80.

16 National Statistics. English indices of deprivation 2015. Ministry of housing clg. National Statistics, 2015.

17 Gregori NZ, Feuer W, Rosenfeld PJ. Novel method for analyzing Snellen visual acuity measurements. Retina 2010;30:1046-50.

18 Nghiem-Buffet $S$, Baillif $S$, Regnier $S$, et al. Treatment patterns of ranibizumab intravitreal injection and dexamethasone intravitreal implant for retinal vein occlusion in the USA. Eye 2017;31:551-9. 